CRYSTALLOGRAPHIC COMMUNICATIONS

ISSN 2056-9890

Received 30 October 2019

Accepted 28 December 2019

Edited by M. Weil, Vienna University of Technology, Austria

Keywords: crystal structure; salts; hydrogen bonds; Hirshfeld surface.

CCDC reference: 1974526

Supporting information: this article has supporting information at journals.iucr.org/e

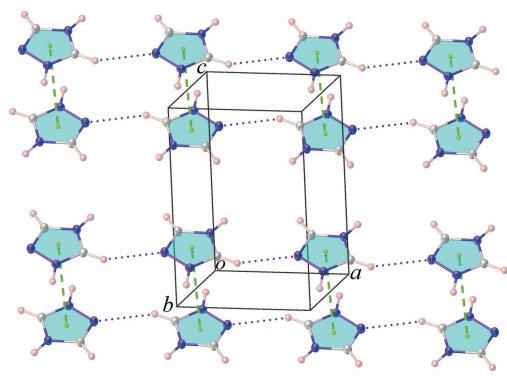

OPEN $\odot$ ACCESS

\section{Crystal structure and Hirshfeld surface analysis of 1,2,4-triazolium hydrogen oxalate}

\author{
Nutcha Ponjan, ${ }^{\mathrm{a}}$ Purita Aroonchat ${ }^{\mathrm{b}}$ and Kittipong Chainok ${ }^{\mathrm{a} *}$ \\ aMaterials and Textile Technology, Faculty of Science and Technology, Thammasat University, Khlong Luang, Pathum \\ Thani, 12121, Thailand, and ${ }^{\mathbf{b}}$ Science Classroom in University-Affiliated School Projects (SCiUS), Suankularb, Wittayalai \\ Rangsit School, Muang, Pathum Thani 12120, Thailand. *Correspondence e-mail: kc@tu.ac.th
}

The asymmetric unit of the title 1:1 salt 1,2,4-triazolium hydrogen oxalate, $\mathrm{C}_{2} \mathrm{H}_{4} \mathrm{~N}_{3}{ }^{+} \cdot \mathrm{C}_{2} \mathrm{HO}_{4}{ }^{-}$(I), comprises one 1,2,4-triazolium cation and one hydrogen oxalate anion. In the crystal, the hydrogen oxalate anions are linked by $\mathrm{O}-$ $\mathrm{H} \cdots \mathrm{O}$ hydrogen bonds into chains running parallel to [100]. In turn, the anionic chains are linked through the 1,2,4-triazolium cations by charge-assisted ${ }^{+} \mathrm{N}-$ $\mathrm{H} \cdots \mathrm{O}^{-}$hydrogen bonds into sheets aligned parallel to $(01 \overline{1})$. The sheets are further stacked through $\pi-\pi$ interactions between the 1,2,4-triazolium rings [centroid-to-centroid distance $=3.642$ (3) $\AA$, normal distance $=3.225$ (3) $\mathrm{A}$, slippage $1.691 \AA$ ] , resulting in the formation of a three-dimensional supramolecular network. Hirshfeld surface analysis of the title salt suggests that the most significant contributions to the crystal packing are by $\mathrm{H} \cdots \mathrm{O} / \mathrm{O} \cdots \mathrm{H}$ and $\mathrm{H} \cdots \mathrm{N} / \mathrm{N} \cdots \mathrm{H}$ contacts involving the hydrogen bonds.

\section{Chemical context}

The oxalate anion $\left(\mathrm{C}_{2} \mathrm{O}_{4}{ }^{2-}\right)$, i.e. the complete deprotonation product of oxalic acid $\left(\mathrm{C}_{2} \mathrm{H}_{2} \mathrm{O}_{4}\right)$, is a small, rigid, planar species and has been widely used as a ligand in the formation of coordination polymers (Gruselle et al., 2006; Abraham et al., 2014). This ligand possesses four electron-donating $\mathrm{O}$ atoms and can display versatile coordination modes upon metal complexation. As a result, a large number of compounds with multi-dimensional coordination networks with short intermetallic distances have been synthesized along with the investigation of interesting properties (Clemente-León et al., 2011). During our synthetic efforts to develop novel lanthanide coordination polymers with rigid, short, organic ligands including the oxalate anion, the title salt $\mathrm{C}_{2} \mathrm{H}_{4} \mathrm{~N}_{3}{ }^{+} \cdot \mathrm{C}_{2} \mathrm{HO}_{4}{ }^{-}$(I) was obtained unexpectedly from the reaction of terbium(III) chloride hexahydrate, oxalic acid, and 1,2,4-triazole in water at room temperature.<smiles>c1n[nH]c[nH+]1</smiles><smiles>O=C(O)C(=O)O</smiles>

Herein, we describe the crystal structure and Hirshfeld surface analysis of the title salt (I).

\section{Structural commentary}

As shown in Fig. 1, the asymmetric unit consists of one 1,2,4triazolium cation and one hydrogen oxalate anion. In the 
Table 1

Hydrogen-bond geometry $\left(\AA,^{\circ}\right)$.

\begin{tabular}{lllll}
\hline$D-\mathrm{H} \cdots A$ & $D-\mathrm{H}$ & $\mathrm{H} \cdots A$ & $D \cdots A$ & $D-\mathrm{H} \cdots A$ \\
\hline $\mathrm{O} 1-\mathrm{H} 1 A \cdots \mathrm{O} 4^{\mathrm{i}}$ & $0.94(2)$ & $1.61(2)$ & $2.5447(13)$ & $175.0(18)$ \\
$\mathrm{N} 1-\mathrm{H} 1 \cdots \mathrm{O} 3$ & $0.91(2)$ & $1.81(2)$ & $2.7199(15)$ & $175.4(18)$ \\
$\mathrm{N} 2-\mathrm{H} 2 \cdots \mathrm{O} 4^{\mathrm{ii}}$ & $0.96(2)$ & $1.80(2)$ & $2.7443(15)$ & $167.3(19)$ \\
$\mathrm{C} 3-\mathrm{H} 3 \cdots \mathrm{O} 2^{\mathrm{iii}}$ & 0.93 & 2.40 & $3.1717(17)$ & 141 \\
$\mathrm{C} 3-\mathrm{H} 3 \cdots 3^{\text {iv }}$ & 0.93 & 2.58 & $3.3939(18)$ & 146 \\
$\mathrm{C} 4-\mathrm{H} 4 \cdots \mathrm{O} 1$ & 0.93 & 2.45 & $3.0289(16)$ & 120 \\
$\mathrm{C} 4-\mathrm{H} 4 \cdots \mathrm{O} 3^{\mathrm{i}}$ & 0.93 & 2.30 & $3.1625(17)$ & 153 \\
\hline
\end{tabular}

Symmetry codes: (i) $x-1, y, z$; (ii) $x-1, y-1, z-1$; (iii) $x, y-1, z-1$; (iv) $x+1, y, z$.

hydrogen oxalate anion, the $\mathrm{C} 1-\mathrm{O} 1$ bond to the $\mathrm{O}$ atom that carries the $\mathrm{H}$ atom is significantly longer [1.3066 (14) $\AA$ ] than the $\mathrm{C} 1-\mathrm{O} 2$ bond [1.1976 (15) $\AA$ ], whereas the $\mathrm{C} 2-\mathrm{O} 3$ and $\mathrm{C} 2-\mathrm{O} 4$ bond lengths of the carboxylate group show intermediate values [1.2370 (15) and 1.2586 (14) $\AA$, respectively]. The hydrogen oxalate molecule is nearly planar with an $\mathrm{O} 2-$ $\mathrm{C} 1-\mathrm{C} 2-\mathrm{O} 4$ torsion angle of $2.3(2)^{\circ}$. The 1,2,4-triazolium molecule is perfectly planar with a root-mean-square (r.m.s.) deviation (excluding hydrogen atoms) of $0.001 \AA$. The cationic and anionic molecules are coplanar with an r.m.s. deviation of $0.020 \AA$.

\section{Supramolecular features}

Extensive hydrogen-bonding interactions in the crystal of the title salt (I) are observed, the numerical values of which are collated in Table 1. As shown in Fig. 2, each hydrogen oxalate anion is linked with another anion by $\mathrm{O}-\mathrm{H} \cdots \mathrm{O}$ hydrogen bonds into an infinite chain running parallel to [100]. The anionic chains are linked by charge-assisted ${ }^{+} \mathrm{N}-\mathrm{H} \cdots \mathrm{O}^{-}$ hydrogen bonds involving the 1,2,4-triazolium cations into sheets extending parallel to $(01 \overline{1})$. Additionally, intrasheet $\mathrm{C}-$ $\mathrm{H} \cdots \mathrm{O}$ hydrogen and $\mathrm{C}-\mathrm{H} \cdots \mathrm{N}$ hydrogen bonds involving the cationic molecules are also observed. The sheets are further stacked through $\pi-\pi$ interactions between the 1,2,4-triazolium rings [centroid-to-centroid distance $=3.642(3) \AA$, normal distance $=3.225$ (3) $\AA$, slippage $1.691 \AA$ ] , Fig. 3, resulting in the formation of a three-dimensional supramolecular network.

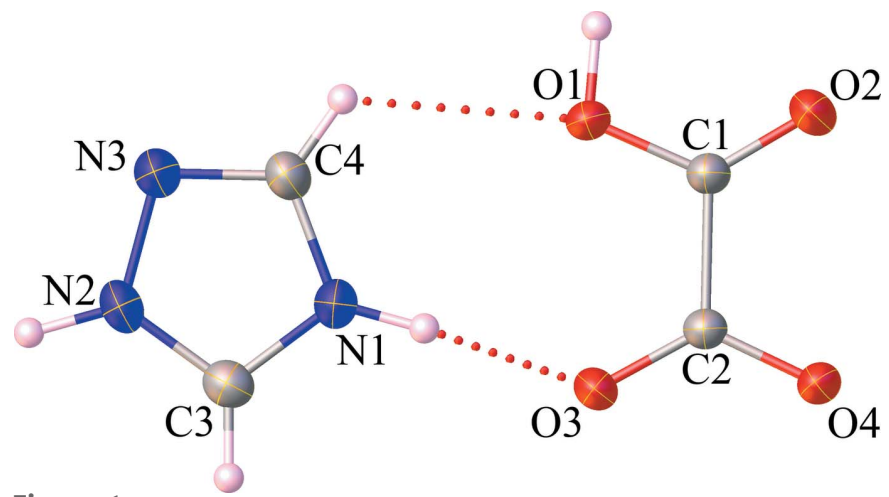

Figure 1

The structures of the molecular entities in the title salt (I) showing the atom-labelling scheme. Displacement ellipsoids are drawn at the 50\% probability level, and hydrogen bonds are shown as dotted lines.

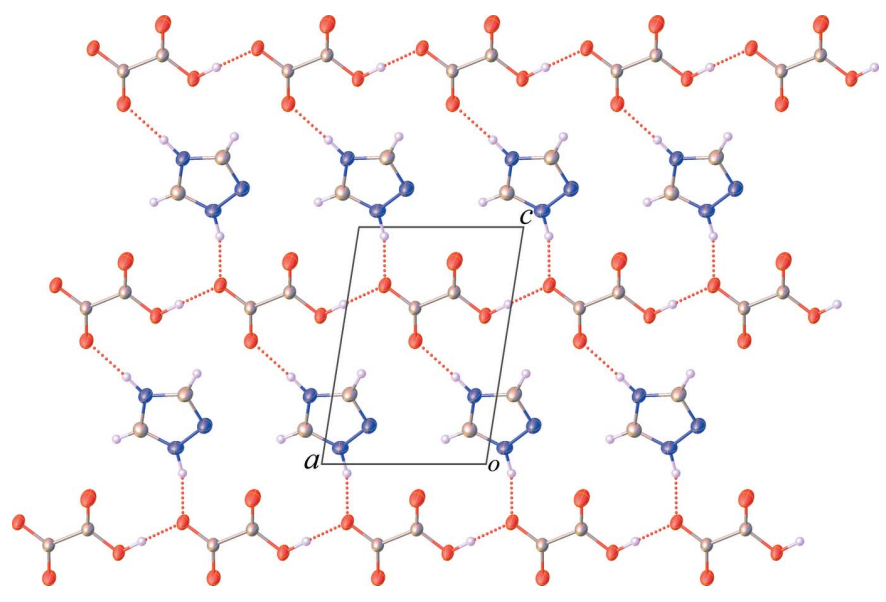

Figure 2

Partial view along [010] of the title salt (I), showing the $\mathrm{O}-\mathrm{H} \cdots \mathrm{O}$ and $\mathrm{N}-\mathrm{H} \cdots \mathrm{O}$ hydrogen-bonded sheet propagating parallel to $(01 \overline{1}) . \mathrm{C}-$ $\mathrm{H} \cdots \mathrm{O}$ and $\mathrm{C}-\mathrm{H} \cdots \mathrm{N}$ hydrogen bonds are omitted for clarity.

\section{Hirshfeld surface analysis}

In order to quantify the nature of the intermolecular interactions present in the crystal structure, Hirshfeld surfaces (McKinnon et al., 2007) and their associated two-dimensional fingerprint plots (Spackman \& McKinnon, 2002) were calculated using CrystalExplorer17 (Turner et al., 2017). The contribution of interatomic contacts to the $d_{\text {norm }}$ surface of the title salt and the individual cations and anions are compared and shown in Fig. 4. In all cases, $\mathrm{H} \cdots \mathrm{O} / \mathrm{O} \cdots \mathrm{H}$ contacts (i.e. ${ }^{+} \mathrm{N}-\mathrm{H} \cdots \mathrm{O}^{-}, \mathrm{O}-\mathrm{H} \cdots \mathrm{O}, \mathrm{C}-\mathrm{H} \cdots \mathrm{O}$ ) were found to be the major contributors towards the Hirshfeld surface, whereas $\mathrm{H} \cdots \mathrm{N} / \mathrm{N} \cdots \mathrm{H}$ contacts (i.e. $\mathrm{C}-\mathrm{H} \cdots \mathrm{N}$ ) between the 1,2,4triazolium cations play a minor role in the stabilization of the crystal packing. The differences between the individual fingerprints of cations and anions result from different distributions of the $\mathrm{C} \cdots \mathrm{N} / \mathrm{N} \cdots \mathrm{C}$ contacts (i.e. $\pi-\pi$ stacking). It was found that the $\mathrm{H} \cdots \mathrm{H}$ contacts have a relatively small contri-

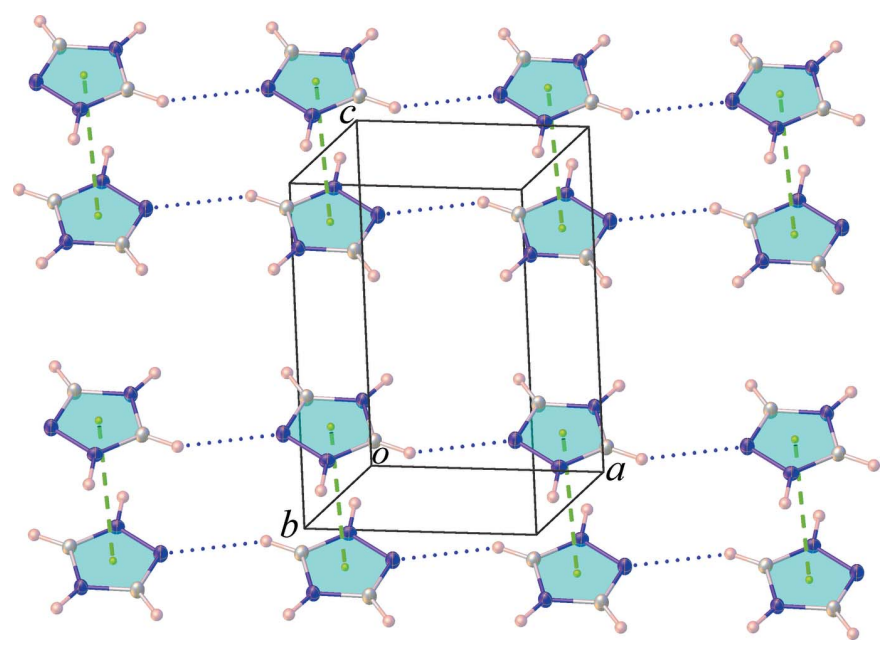

Figure 3

A view of the $\pi-\pi$ stacking interactions along with the $\mathrm{C}-\mathrm{H} \cdots \mathrm{N}$ hydrogen bonds in the title salt (I). 


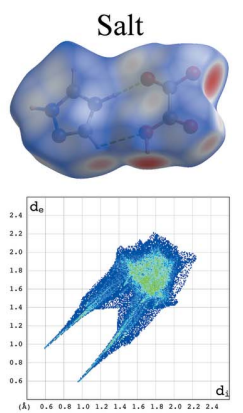

(a) All Salt

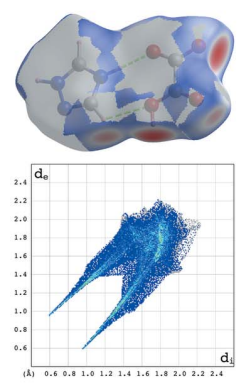

(d) $\mathrm{H} \cdots \mathrm{O} / \mathrm{O} \cdots \mathrm{H}(52.7 \%)$
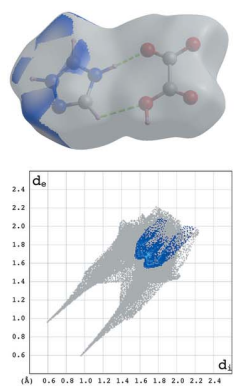

(g) $\mathrm{C} \cdots \mathrm{N} / \mathrm{N} \cdots \mathrm{C}(5.3 \%)$

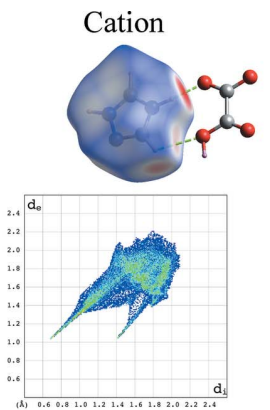

(b) All Cation

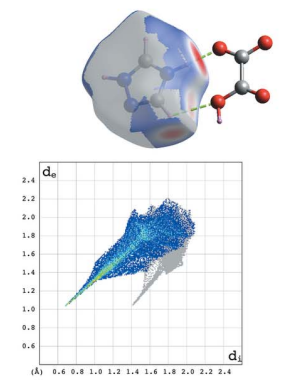

(e) $\mathrm{H} \cdots \mathrm{O} / \mathrm{O} \cdots \mathrm{H}(45.7 \%)$

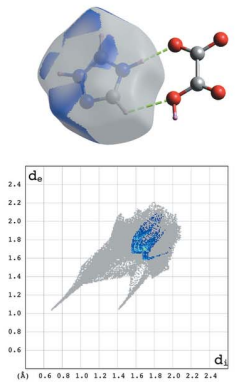

(h) $\mathrm{C} \cdots \mathrm{N} / \mathrm{N} \cdots \mathrm{C}(6.0 \%)$

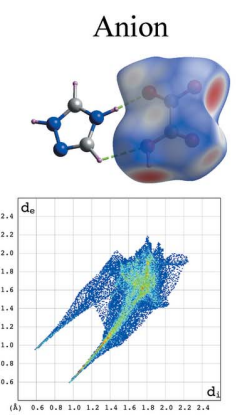

(c) All Anion

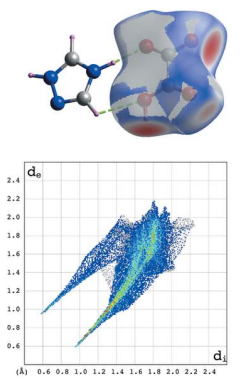

(f) $\mathrm{H} \cdots \mathrm{O} / \mathrm{O} \cdots \mathrm{H}(71.6 \%)$
Figure 4

Full two-dimensional fingerprint plots of the title salt (I) $(a)$, and its cation $(b)$ and anion $(c)$; separate contact types for the salt are given in $(d)-(h)$ with relative contributions. Hirshfeld surfaces mapped over $d_{\text {norm }}$ are displayed in all plots.

bution of only $7.7 \%$ to the entire Hirshfeld surfaces of the title salt.

\section{Database survey}

A search of the Cambridge Structural Database (CSD version 5.40, August 2019 update; Groom et al., 2016) for structures with hydrogen oxalate gave 666 hits of which five are hydrogen-bonded salts of triazolium, viz. AFIVAO (Essid et al., 2013) and CIRXEH (Matulková et al., 2008), or imidazolium, viz. EVAPEX (Zhu, 2011), MEQPAZ (MacDonald et al., 2001) and MEQPAZ01 (Prasad et al., 2002).

\section{Synthesis and crystallization}

An aqueous solution $(5 \mathrm{ml})$ of oxalic acid $(0.09 \mathrm{~g}, 0.01 \mathrm{~mol})$ and 1,2,4-triazole $(0.07 \mathrm{~g}, 0.01 \mathrm{mmol})$ was added dropwise to
Table 2

Experimental details.

Crystal data

Chemical formula

$M_{\mathrm{r}}$

Crystal system, space group

Temperature (K)

$a, b, c(\AA)$

$\alpha, \beta, \gamma\left({ }^{\circ}\right)$

$V\left(\AA^{3}\right)$

Z

Radiation type

$\mu\left(\mathrm{mm}^{-1}\right)$

Crystal size (mm)

$\mathrm{C}_{2} \mathrm{H}_{4} \mathrm{~N}_{3}{ }^{+} \cdot \mathrm{C}_{2} \mathrm{HO}_{4}{ }^{-}$

159.11

Triclinic, $P \overline{1}$

296

5.592 (1), 7.2162 (12), 8.4021 (13)

109.148 (6), 93.889 (7), 103.282 (6)

307.92 (9)

Mo $K \alpha$

0.16

$0.34 \times 0.22 \times 0.22$

Data collection

Diffractometer

Absorption correction

$T_{\min }, T_{\max }$

No. of measured, independent and observed $[I>2 \sigma(I)]$ reflections

$R_{\text {int }}$

$(\sin \theta / \lambda)_{\max }\left(\AA^{-1}\right)$

Bruker D8 Quest CMOS
PHOTON II
Multi-scan (SADABS; Bruker,
2016)
$0.638,0.746$
$4805,1524,1278$
0.038
0.668

$0.037,0.102,1.07$
1524
112
H atoms treated by a mixture of
independent and constrained
refinement
$0.37,-0.20$

Refinement

$R\left[F^{2}>2 \sigma\left(F^{2}\right)\right], w R\left(F^{2}\right), S$

No. of reflections

No. of parameters

$\mathrm{H}$-atom treatment

$\Delta \rho_{\max }, \Delta \rho_{\min }\left(\mathrm{e} \AA^{-3}\right)$

$0.37,-0.20$

Computer programs: APEX3 and SAINT (Bruker, 2016), SHELXT (Sheldrick, 2015a), SHELXL (Sheldrick, 2015b) and OLEX2 (Dolomanov et al., 2009).

an aqueous solution $(5 \mathrm{ml})$ of $\mathrm{TbCl}_{3} \cdot 6 \mathrm{H}_{2} \mathrm{O}(0.37 \mathrm{~g}, 0.01 \mathrm{~mol})$ under constant stirring for one h. The resulting solution was filtered to remove any undissolved solid. The filtrate was allowed to slowly evaporate at room temperature. After two weeks, colourless block-shaped crystals of the title salt (I) suitable for X-ray analysis were obtained.

\section{Refinement}

Crystal data, data collection and structure refinement details are summarized in Table 2. The carboxyl and triazolium $\mathrm{H}$ atoms were located in difference-Fourier maps and were freely refined. Carbon-bound $\mathrm{H}$ atoms were placed in calculated positions and refined using a riding-model approximation with $\mathrm{C}-\mathrm{H}=0.93 \AA$ and $U_{\text {iso }}(\mathrm{H})=1.2 U_{\text {eq }}(\mathrm{C})$.

\section{Acknowledgements}

The authors thank the Faculty of Science and Technology, Thammasat University, for funds to purchase the X-ray diffractometer.

\section{Funding information}

Funding for this research was provided by: The National Research Council of Thailand grant provided by the Thammasat University (grant No. 4/2561). 


\section{References}

Abraham, F., Arab-Chapelet, B., Rivenet, M., Tamain, C. \& Grandjean, S. (2014). Coord. Chem. Rev. 266-267, 28-68.

Bruker (2016). APEX3, SAINT and SADABS. Bruker AXS Inc., Madison, Wisconsin, USA.

Clemente-León, M., Coronado, E., Martí-Gastaldo, C. \& Romero, F. M. (2011). Chem. Soc. Rev. 40, 473-497.

Dolomanov, O. V., Bourhis, L. J., Gildea, R. J., Howard, J. A. K. \& Puschmann, H. (2009). J. Appl. Cryst. 42, 339-341.

Essid, M., Marouani, H., Al-Deyab, S. S. \& Rzaigui, M. (2013). Acta Cryst. E69, o1279.

Groom, C. R., Bruno, I. J., Lightfoot, M. P. \& Ward, S. C. (2016). Acta Cryst. B72, 171-179.

Gruselle, M., Train, C., Boubekeur, K., Gredin, P. \& Ovanesyan, N. (2006). Coord. Chem. Rev. 250, 2491-2500.
MacDonald, J. C., Dorrestein, P. C. \& Pilley, M. M. (2001). Cryst. Growth Des. 1, 29-38.

Matulková, I., Němec, I., Teubner, K., Němec, P. \& Mička, Z. (2008). J. Mol. Struct. 873, 46-60.

McKinnon, J. J., Jayatilaka, D. \& Spackman, M. A. (2007). Chem. Commun. pp. 3814-3816.

Prasad, R. A., Neeraj, S., Vaidhyanathan, R. \& Natarajan, S. (2002). J. Solid State Chem. 166, 128-141.

Sheldrick, G. M. (2015a). Acta Cryst. A71, 3-8.

Sheldrick, G. M. (2015b). Acta Cryst. C71, 3-8.

Spackman, M. A. \& McKinnon, J. J. (2002). CrystEngComm, 4, 378392.

Turner, M. J., Mckinnon, J. J., Wolff, S. K., Grimwood, D. J., Spackman, P. R., Jayatilaka, D. \& Spackman, M. A. (2017). CrystalExplorer17. The University of Western Australia.

Zhu, R.-Q. (2011). Acta Cryst. E67, o1627. 


\section{supporting information}

Acta Cryst. (2020). E76, 137-140 [https://doi.org/10.1107/S2056989019017304]

\section{Crystal structure and Hirshfeld surface analysis of 1,2,4-triazolium hydrogen oxalate}

\section{Nutcha Ponjan, Purita Aroonchat and Kittipong Chainok}

Computing details

Data collection: APEX3 (Bruker, 2016); cell refinement: SAINT (Bruker, 2016); data reduction: SAINT (Bruker, 2016); program(s) used to solve structure: SHELXT (Sheldrick, 2015a); program(s) used to refine structure: SHELXL (Sheldrick, 2015b); molecular graphics: OLEX2 (Dolomanov et al., 2009); software used to prepare material for publication: OLEX2 (Dolomanov et al., 2009).

1,2,4-Triazolium hydrogen oxalate

\section{Crystal data}

$\mathrm{C}_{2} \mathrm{H}_{4} \mathrm{~N}_{3}{ }^{+} \cdot \mathrm{C}_{2} \mathrm{HO}_{4}^{-}$

$M_{r}=159.11$

Triclinic, $P \overline{1}$

$a=5.592(1) \AA$

$b=7.2162(12) \AA$

$c=8.4021(13) \AA$

$\alpha=109.148(6)^{\circ}$

$\beta=93.889(7)^{\circ}$

$\gamma=103.282(6)^{\circ}$

$V=307.92(9) \AA^{3}$

\section{Data collection}

Bruker D8 Quest CMOS PHOTON II diffractometer

Radiation source: sealed x-ray tube Graphite monochromator

Detector resolution: 7.39 pixels $\mathrm{mm}^{-1}$

$\omega$ and $\varphi$ scans

Absorption correction: multi-scan

(SADABS; Bruker, 2016)

$T_{\min }=0.638, T_{\max }=0.746$

\section{Refinement}

Refinement on $F^{2}$

Least-squares matrix: full

$R\left[F^{2}>2 \sigma\left(F^{2}\right)\right]=0.037$

$w R\left(F^{2}\right)=0.102$

$S=1.07$

1524 reflections

112 parameters

0 restraints
$Z=2$

$F(000)=164$

$D_{\mathrm{x}}=1.716 \mathrm{Mg} \mathrm{m}^{-3}$

Mo $K \alpha$ radiation, $\lambda=0.71073 \AA$

Cell parameters from 2650 reflections

$\theta=2.6-28.3^{\circ}$

$\mu=0.16 \mathrm{~mm}^{-1}$

$T=296 \mathrm{~K}$

Block, light colourless

$0.34 \times 0.22 \times 0.22 \mathrm{~mm}$

4805 measured reflections

1524 independent reflections

1278 reflections with $I>2 \sigma(I)$

$R_{\text {int }}=0.038$

$\theta_{\text {max }}=28.4^{\circ}, \theta_{\min }=2.6^{\circ}$

$h=-6 \rightarrow 7$

$k=-9 \rightarrow 8$

$l=-11 \rightarrow 11$

Hydrogen site location: mixed

$\mathrm{H}$ atoms treated by a mixture of independent and constrained refinement

$w=1 /\left[\sigma^{2}\left(F_{\mathrm{o}}^{2}\right)+(0.0441 P)^{2}+0.0686 P\right]$

where $P=\left(F_{\mathrm{o}}^{2}+2 F_{\mathrm{c}}^{2}\right) / 3$

$(\Delta / \sigma)_{\max }<0.001$

$\Delta \rho_{\max }=0.37 \mathrm{e} \AA^{-3}$

$\Delta \rho_{\min }=-0.20 \mathrm{e} \AA^{-3}$ 


\section{Special details}

Geometry. All esds (except the esd in the dihedral angle between two 1.s. planes) are estimated using the full covariance matrix. The cell esds are taken into account individually in the estimation of esds in distances, angles and torsion angles; correlations between esds in cell parameters are only used when they are defined by crystal symmetry. An approximate (isotropic) treatment of cell esds is used for estimating esds involving l.s. planes.

Fractional atomic coordinates and isotropic or equivalent isotropic displacement parameters $\left(\hat{A}^{2}\right)$

\begin{tabular}{lllll}
\hline & $x$ & $y$ & $z$ & $U_{\text {iso }} * U_{\text {eq }}$ \\
\hline O1 & $0.14792(16)$ & $0.88282(15)$ & $0.62587(12)$ & $0.0335(3)$ \\
H1A & $0.020(4)$ & $0.935(3)$ & $0.674(2)$ & $0.069(6)^{*}$ \\
O2 & $0.38612(19)$ & $1.12875(18)$ & $0.85543(14)$ & $0.0506(3)$ \\
O3 & $0.54794(17)$ & $0.76994(15)$ & $0.51985(12)$ & $0.0365(3)$ \\
O4 & $0.78723(16)$ & $1.00344(15)$ & $0.75427(12)$ & $0.0345(3)$ \\
N1 & $0.1358(2)$ & $0.53625(17)$ & $0.28813(14)$ & $0.0305(3)$ \\
H1 & $0.270(4)$ & $0.620(3)$ & $0.367(2)$ & $0.057(5)^{*}$ \\
N2 & $-0.0976(2)$ & $0.30879(17)$ & $0.06817(14)$ & $0.0305(3)$ \\
H2 & $-0.162(4)$ & $0.201(3)$ & $-0.039(3)$ & $0.063(6)^{*}$ \\
N3 & $-0.2527(2)$ & $0.40637(18)$ & $0.16122(14)$ & $0.0352(3)$ \\
C1 & $0.3594(2)$ & $0.98777(18)$ & $0.72504(15)$ & $0.0264(3)$ \\
C2 & $0.5818(2)$ & $0.91101(18)$ & $0.65830(15)$ & $0.0249(3)$ \\
C3 & $0.1335(2)$ & $0.38679(19)$ & $0.14421(16)$ & $0.0304(3)$ \\
H3 & 0.270783 & 0.345169 & 0.104695 & $0.036^{*}$ \\
C4 & $-0.1040(2)$ & $0.5435(2)$ & $0.29349(17)$ & $0.0340(3)$ \\
H4 & -0.155357 & 0.635686 & 0.381391 & $0.041^{*}$ \\
& & & & \\
\hline
\end{tabular}

Atomic displacement parameters $\left(\AA^{2}\right)$

\begin{tabular}{lllllll}
\hline & $U^{11}$ & $U^{22}$ & $U^{33}$ & $U^{12}$ & $U^{13}$ & $U^{23}$ \\
\hline $\mathrm{O} 1$ & $0.0174(4)$ & $0.0398(5)$ & $0.0303(5)$ & $0.0095(4)$ & $0.0014(4)$ & $-0.0054(4)$ \\
$\mathrm{O} 2$ & $0.0280(5)$ & $0.0571(7)$ & $0.0392(6)$ & $0.0155(5)$ & $-0.0002(4)$ & $-0.0201(5)$ \\
$\mathrm{O} 3$ & $0.0224(5)$ & $0.0396(5)$ & $0.0306(5)$ & $0.0093(4)$ & $0.0027(4)$ & $-0.0101(4)$ \\
$\mathrm{O} 4$ & $0.0183(4)$ & $0.0414(5)$ & $0.0294(5)$ & $0.0093(4)$ & $-0.0003(4)$ & $-0.0064(4)$ \\
$\mathrm{N} 1$ & $0.0231(5)$ & $0.0316(6)$ & $0.0269(5)$ & $0.0052(4)$ & $-0.0002(4)$ & $-0.0003(4)$ \\
$\mathrm{N} 2$ & $0.0319(6)$ & $0.0286(5)$ & $0.0225(5)$ & $0.0072(4)$ & $0.0021(4)$ & $-0.0009(4)$ \\
$\mathrm{N} 3$ & $0.0264(6)$ & $0.0380(6)$ & $0.0294(6)$ & $0.0091(5)$ & $0.0012(5)$ & $-0.0029(5)$ \\
$\mathrm{C} 1$ & $0.0190(6)$ & $0.0304(6)$ & $0.0236(6)$ & $0.0083(5)$ & $0.0023(4)$ & $0.0004(5)$ \\
$\mathrm{C} 2$ & $0.0183(5)$ & $0.0274(6)$ & $0.0238(6)$ & $0.0071(4)$ & $0.0033(4)$ & $0.0018(5)$ \\
$\mathrm{C} 3$ & $0.0281(6)$ & $0.0313(6)$ & $0.0285(6)$ & $0.0103(5)$ & $0.0059(5)$ & $0.0046(5)$ \\
$\mathrm{C} 4$ & $0.0273(7)$ & $0.0359(7)$ & $0.0275(6)$ & $0.0101(5)$ & $0.0014(5)$ & $-0.0038(5)$ \\
\hline
\end{tabular}

Geometric parameters $\left(\AA,{ }^{\circ}\right)$

\begin{tabular}{llll}
\hline $\mathrm{O} 1-\mathrm{H} 1 \mathrm{~A}$ & $0.94(2)$ & $\mathrm{N} 2-\mathrm{H} 2$ & $0.96(2)$ \\
$\mathrm{O} 1-\mathrm{C} 1$ & $1.3066(14)$ & $\mathrm{N} 2-\mathrm{N} 3$ & $1.3677(16)$ \\
$\mathrm{O} 2-\mathrm{C} 1$ & $1.1976(15)$ & $\mathrm{N} 2-\mathrm{C} 3$ & $1.3089(17)$ \\
$\mathrm{O} 3-\mathrm{C} 2$ & $1.2370(15)$ & $\mathrm{N} 3-\mathrm{C} 4$ & $1.2967(17)$ \\
$\mathrm{O} 4-\mathrm{C} 2$ & $1.2586(14)$ & $\mathrm{C} 1-\mathrm{C} 2$ & $1.5413(17)$
\end{tabular}




$\begin{array}{llll}\mathrm{N} 1-\mathrm{H} 1 & 0.91(2) & \mathrm{C} 3-\mathrm{H} 3 & 0.9300 \\ \mathrm{~N} 1-\mathrm{C} 3 & 1.3272(16) & \mathrm{C} 4-\mathrm{H} 4 & 0.9300 \\ \mathrm{~N} 1-\mathrm{C} 4 & 1.3568(18) & & \\ \mathrm{C} 1-\mathrm{O} 1-\mathrm{H} 1 \mathrm{~A} & 109.1(12) & \mathrm{O} 2-\mathrm{C} 1-\mathrm{C} 2 & 121.49(11) \\ \mathrm{C} 3-\mathrm{N} 1-\mathrm{H} 1 & 127.8(13) & \mathrm{O} 3-\mathrm{C} 2-\mathrm{O} 4 & 125.86(11) \\ \mathrm{C} 3-\mathrm{N} 1-\mathrm{C} 4 & 106.09(11) & \mathrm{O} 3-\mathrm{C} 2-\mathrm{C} 1 & 119.53(11) \\ \mathrm{C} 4-\mathrm{N} 1-\mathrm{H} 1 & 126.1(13) & \mathrm{O} 4-\mathrm{C} 2-\mathrm{C} 1 & 126.3 \\ \mathrm{~N} 3-\mathrm{N} 2-\mathrm{H} 2 & 120.4(13) & \mathrm{N} 1-\mathrm{C} 3-\mathrm{H} 3 & 107.36(12) \\ \mathrm{C} 3-\mathrm{N} 2-\mathrm{H} 2 & 128.5(13) & \mathrm{N} 2-\mathrm{C} 3-\mathrm{N} 1 & 126.3 \\ \mathrm{C} 3-\mathrm{N} 2-\mathrm{N} 3 & 111.11(11) & \mathrm{N} 2-\mathrm{C} 3-\mathrm{H} 3 & 124.1 \\ \mathrm{C} 4-\mathrm{N} 3-\mathrm{N} 2 & 103.62(11) & \mathrm{N} 1-\mathrm{C} 4-\mathrm{H} 4 & 12.81(12) \\ \mathrm{O} 1-\mathrm{C} 1-\mathrm{C} 2 & 112.90(10) & \mathrm{N} 3-\mathrm{C} 4-\mathrm{N} 1 & 0.08(15) \\ \mathrm{O} 2-\mathrm{C} 1-\mathrm{O} 1 & 125.61(12) & \mathrm{N} 3-\mathrm{C} 4-\mathrm{H} 4 & 0.23(17) \\ & & & 0.06(15) \\ \mathrm{O} 1-\mathrm{C} 1-\mathrm{C} 2-\mathrm{O} 3 & 3.15(18) & \mathrm{N} 3-\mathrm{N} 2-\mathrm{C} 3-\mathrm{N} 1 & -0.18(15) \\ \mathrm{O} 1-\mathrm{C} 1-\mathrm{C} 2-\mathrm{O} 4 & -177.26(11) & \mathrm{C} 3-\mathrm{N} 1-\mathrm{C} 4-\mathrm{N} 3 & \\ \mathrm{O} 2-\mathrm{C} 1-\mathrm{C} 2-\mathrm{O} 3 & -177.27(13) & \mathrm{C} 3-\mathrm{N} 2-\mathrm{N} 3-\mathrm{C} 4 & \mathrm{C} 4-\mathrm{N} 1-\mathrm{C} 3-\mathrm{N} 2 \\ \mathrm{O} 2-\mathrm{C} 1-\mathrm{C} 2-\mathrm{O} 4 & 2.3(2) & & \\ \mathrm{N} 2-\mathrm{N} 3-\mathrm{C} 4-\mathrm{N} 1 & -0.18(16) & & \end{array}$

Hydrogen-bond geometry $\left(\AA,{ }^{\circ}\right)$

\begin{tabular}{lllll}
\hline$D-\mathrm{H} \cdots A$ & $D-\mathrm{H}$ & $\mathrm{H} \cdots A$ & $D \cdots A$ & $D-\mathrm{H} \cdots A$ \\
\hline $\mathrm{O} 1-\mathrm{H} 1 A \cdots \mathrm{O} 4^{\mathrm{i}}$ & $0.94(2)$ & $1.61(2)$ & $2.5447(13)$ & $175.0(18)$ \\
$\mathrm{N} 1-\mathrm{H} 1 \cdots \mathrm{O} 3$ & $0.91(2)$ & $1.81(2)$ & $2.7199(15)$ & $175.4(18)$ \\
$\mathrm{N} 2-\mathrm{H} 2 \cdots \mathrm{O} 4^{\mathrm{ii}}$ & $0.96(2)$ & $1.80(2)$ & $2.7443(15)$ & $167.3(19)$ \\
$\mathrm{C} 3-\mathrm{H} 3 \cdots \mathrm{O} 2^{\mathrm{iii}}$ & 0.93 & 2.40 & $3.1717(17)$ & 141 \\
$\mathrm{C} 3-\mathrm{H} 3 \cdots \mathrm{N} 3^{\text {iv }}$ & 0.93 & 2.58 & $3.3939(18)$ & 146 \\
$\mathrm{C} 4-\mathrm{H} 4 \cdots \mathrm{O} 1$ & 0.93 & 2.45 & $3.0289(16)$ & 120 \\
$\mathrm{C} 4-\mathrm{H} 4 \cdots \mathrm{O} 3^{\mathrm{i}}$ & 0.93 & 2.30 & $3.1625(17)$ & 153
\end{tabular}

Symmetry codes: (i) $x-1, y, z$; (ii) $x-1, y-1, z-1$; (iii) $x, y-1, z-1$; (iv) $x+1, y, z$. 